\title{
Autocorrelation and Crosscorrelation of Electrocardiographic Record during Ventricular Fibrillation
}

\author{
Sintaro KOYAMA, M.D., ${ }^{*}$ Kenichi HARUMI, M.D., ** \\ Keigo SUZUKI, M.D., * Hiroshi WATANABE, M.D., * \\ and Nobumitsu TAKAHASHI, M.D.*
}

The natural course of ventricular fibrillation induced in dogs was analysed by the use of autocorrelation and crosscorrelation function. In the extremity leads, a regularity of fibrillation waves was found until 2 to 5 minutes after the onset of the fibrillation. In the autocorrelogram of the diredt ventricular lead, however, periodic signal was obtained throughout the course of the fibrillation, with varying cycle of the periodic function. Periodic signal was observed only at the onset of fibrillation in the crosscorrelation of the standard leads and also of the right and left ventricular leads. The significance of these findings was discussed.

$\mathrm{T}$ HE natural course of ventricular fibrillation was divided into 4 stages by Wiggers, $\left.{ }^{1)}, 2\right)$ who suggested that the degree of asynchronism of its waves might vary at each stage. Angelakos and Shepherd ${ }^{3)}$ reported that autocorrelation might be a useful tool for the accurate analysis of the various types of ventricular fibrillation in different conditions. The following experiments were carried out to re-evaluate their findings.

\section{METHOD}

Five dogs were anesthetized with nembutal. The electrocardiogram of extremity lead was taken and the ventriculogram of the direct lead was obtained from 2 or 3 points of left and right ventricles in the open chest dog. Ventricular fibrillation was induced by the use of alternating current through external or direct electrodes. The electrocardiogram of the fibrillation was recorded on the Magnetic Recorder ${ }^{+}$and auto- and crosscorrelation functions were obtained by Automatic Analogue Correlator.+ ${ }^{+}$In the correlogram each recorded line

From the Cardiovascular Institute, Tokyo* and the Second Department of Internal Medicine, Faculty of Medicine, University of Tokyo, Tokyo.**

+ Data Recorder, Model MA-23, Sony Corp., Tokyo, Japan.

++ Model CCA-22, Sony Corp., Tokyo, Japan. 
represents correlation function over the entire record (47 seconds duration). The height of the line represents the relative magnitude of the function. The delay time $(\tau)$ is shown on the abscissa, each large interval on the record representing $0.28 \mathrm{sec}$, and $\Delta \tau$ is $10 \mathrm{msec}$.

\section{RESULT}

\section{1) Ventricular fibrillation curves of the extremity lead}

During 2 minutes after the onset of the ventricular fibrillation, a periodic component was observed in the auto- and crosscorrelation of both Lead I and II. Three to 5 minutes after, the periodic signal of

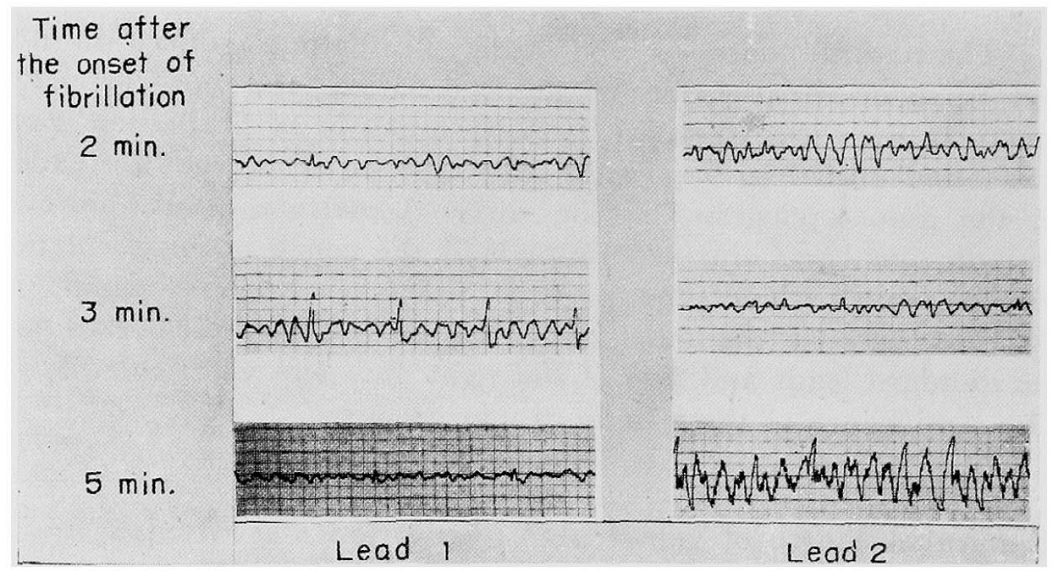

Fig. 1-a. Electrocardiogram of extremity lead during the course of ventricular fibrillation.

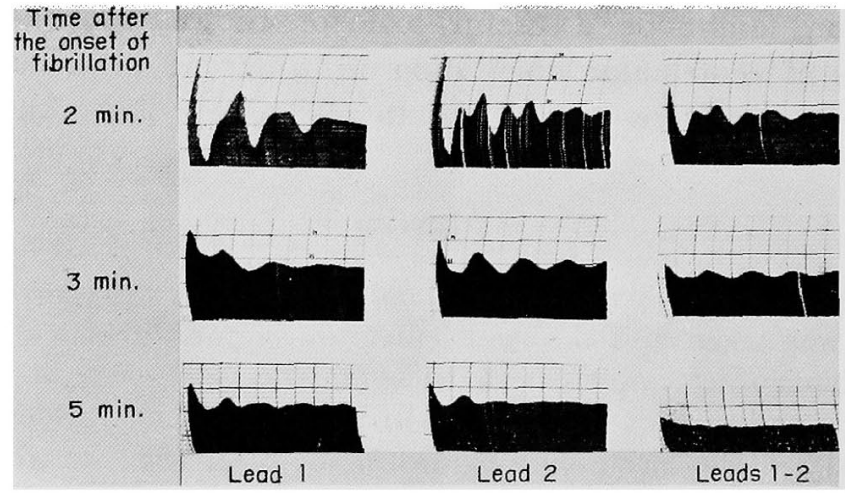

Fig. 1-b. Auto- (left and middle column) and crosscorrelogram (right column) of the extremity lead during the course of ventricular fibrillation.

autocorrelation became relatively obscure as seen in Fig. 1-b. Fig. 1-a shows the electrocardiogram of extremity lead comparable to Fig. 1-b. In 
another case, Lead I contained no regular periodic signal after 5 minutes, but Lead II showed definite regularity of autocorrelation function (Fig. 2).

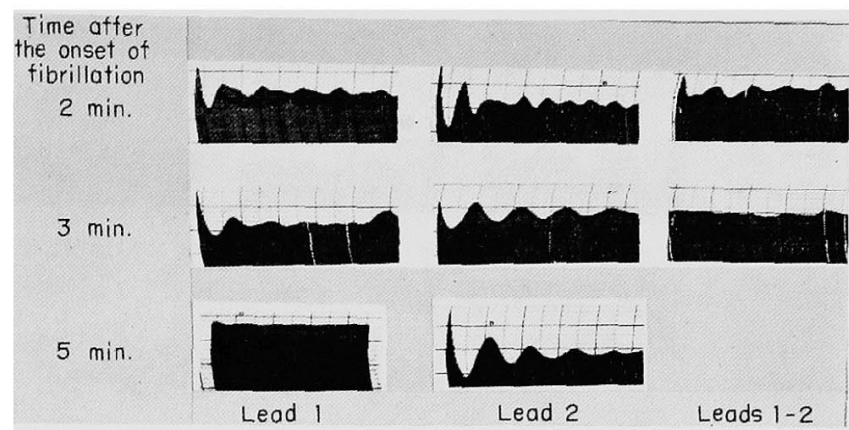

Fig. 2. Auto- (left and middle column) and crosscorrelogram (right column) of the extremity lead during the course of ventricular fibrillation.

2) Ventricular fibrillation curves of the direct lead

At the onset of ventricular fibrillation a periodic signal could be recognized in the autocorrelation of the left and right ventricles and in the crosscorrelation between them. In the autocorrelogram of the left ventricle, a periodic signal became obscure during 3 to 7 minutes and was detected after 11 minutes in the course of the ventricular fibrillation. In the autocorrelogram of the right ventricle, the cycle of periodic com-

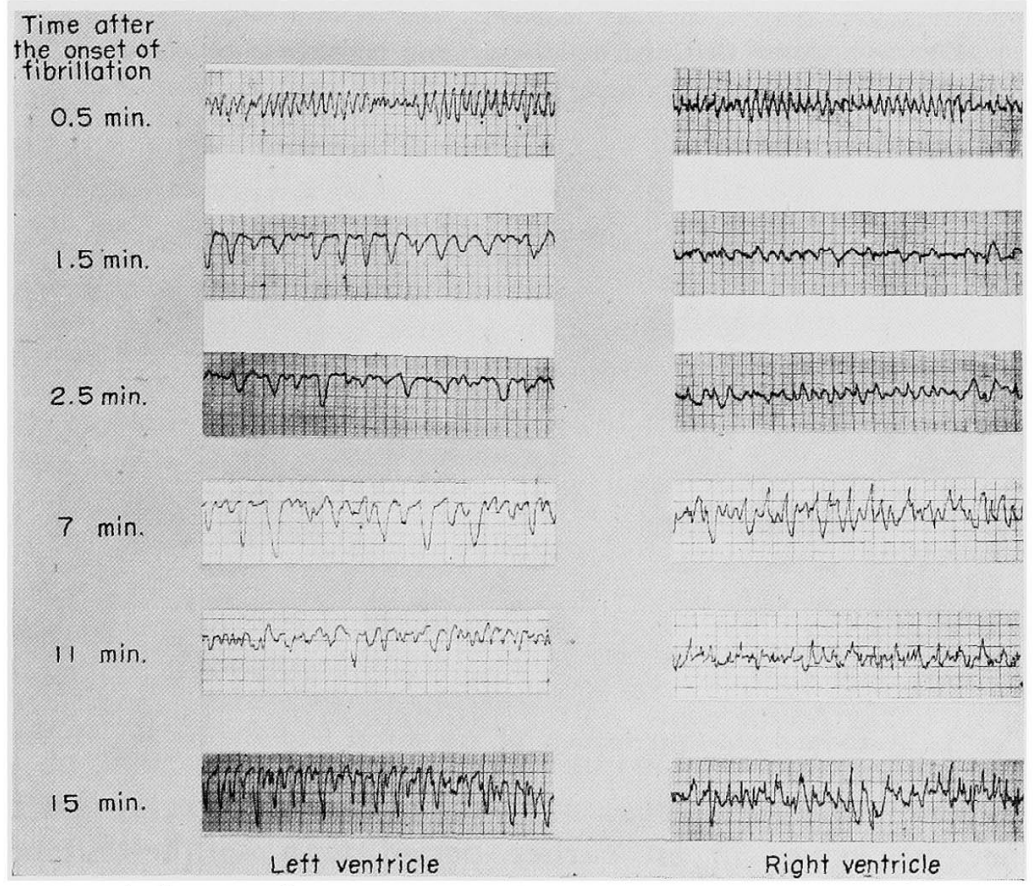

Fig. 3-a. Ventriculogram of the direct lead during the course of ventricular fibrillation. 


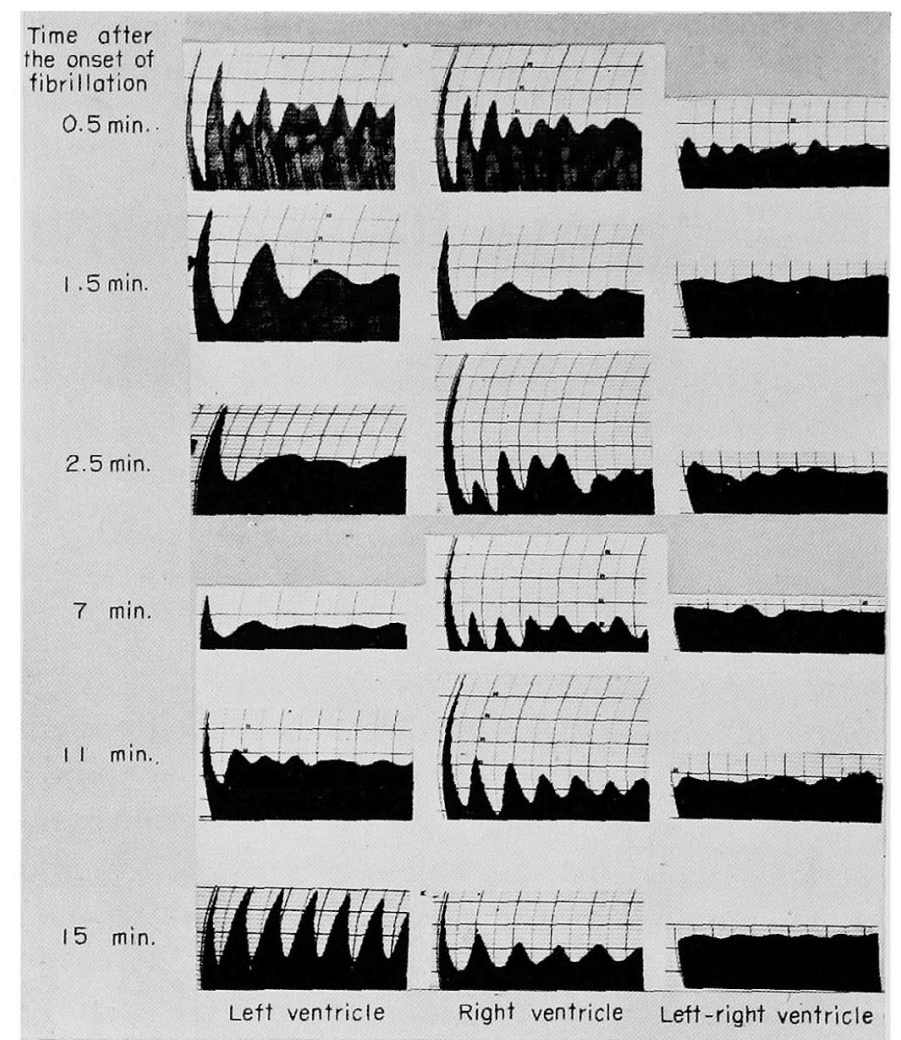

Fig. 3-b. Auto- (left" and middle column) and crosscorrelogram (right column) of the direct lead during the course of ventricular fibrillation.

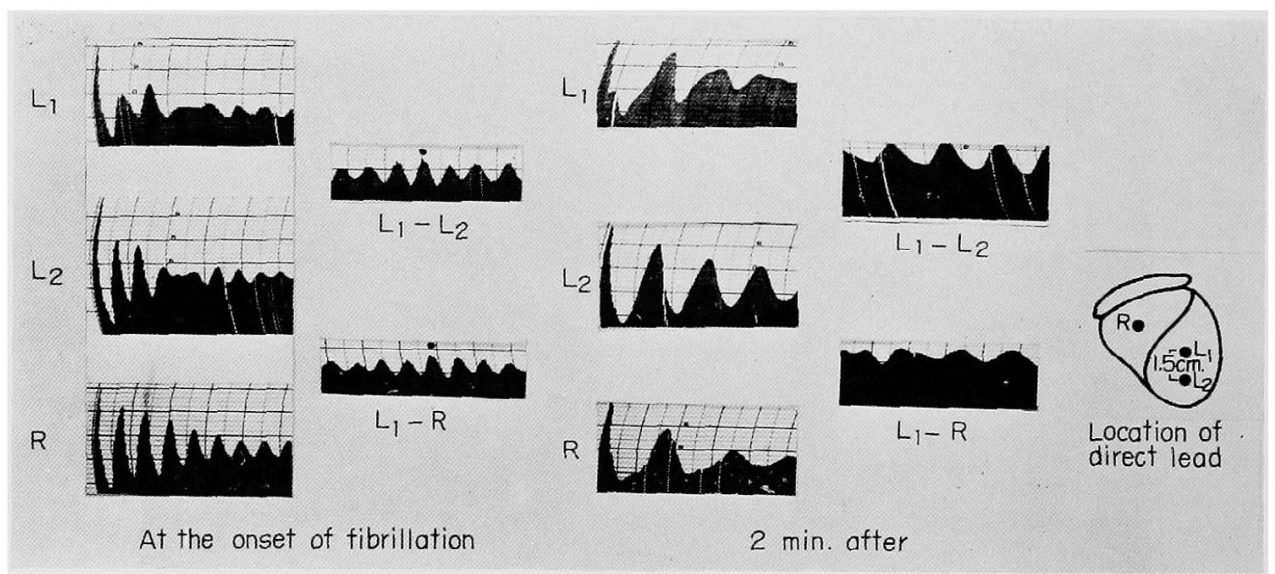

Fig. 4. Auto- and crosscorrelogram of the direct lcad during the course of ventricular fibrillation.

ponent became longer in an earlier period than in the left ventricle. Grosscorrelation of right and left ventriculograms was always random ex- 
cept at the onset of the ventricular fibrillation (Fig. 3-a and b). When the 2 points were placed $1.5 \mathrm{~cm}$. apart from each other on the surface of the left ventricle, periodic component was observed in crosscorrelation between them even after 2 or 3 minutes (Fig. 4).

\section{Discussion}

Wiggers has divided the course of the ventricular fibrillation into 4 stages, namely: a) Undulatory stage, b) Stage of convulsive undulation, c) Stage of tremulous incoordination, and d) Stage of atonic fibrillation, and thought that the latter 2 stages correspond to myocardial anoxia. Angelakos and Shepherd found autocorrelation function periodic only in the first 2 or 3 minutes course of the ventricular fibrillation.

We obtained the same results in Lead $I$ as those by Angelakos and Shepherd, but in Lead II a periodic signal continued for 5 minutes or longer than their results. A periodic function was observed in crosscorrelation between Lead I and Lead II during the 2 minutes after the onset of fibrillation. However, it was interesting to note that a periodic signal was apparent in autocorrelation of the direct left and right ventricular leads throughout the experiments, but not observed in the crosscorrelation except at the onset of fibrillation.

These results suggest that regularity exists in the waves of the ventricular fibrillation almost constantly, and that at the onset a relatively large area of the ventricle was affected by the cycle of a periodic signal appearing in auto- and crosscorrelation. This may be the phase of undulatory and convulsive stages according to Wiggers. The phase during which the periodic signal was observed only in the direct leads may correspond to the stage of tremulous incoordination. In this phase it may be that the contracting area can be divided into smaller sections. It may be that the cycle of a periodic function of a direct lead can rule the smaller area and, therefore, the signal of the direct lead may possibly not be reflected to the extremity leads.

\section{Summary}

(1) Auto- and crosscorrelation of the electrocardiographic record during experimental ventricular fibrillation were investigated in dogs.

(2) At the onset of the ventricular fibrillation a periodic function was observed in the extremity leads and the regularity of the wave vanished earlier in Lead I than Lead II.

(3) In the direct leads the wave of the ventricular fibrillation 
274 KOYAMA, HARUMI, SUZUKI, WATANABE, AND TAKAHASHI $\underset{\text { May, }}{\stackrel{\text { Hart } J .}{1962}}$ included the regular component which was indicated by periodicity in the auto- and crosscorrelation. Except at the onset of fibrillation there was no relation between the waves of both ventricles, as shown by the random function in crosscorrelation.

\section{REFERENCES}

1. Wiggers, C. J.: Am. Heart J. 5: 351, 1930.

2. Wiggers, C. J.: Am. Heart J. 20 : 399, 1940.

3. Angelakos, E. T. and Shepherd, G. M.: Girculat. Res, 5: 657, 1957.

4. Lee, Y, W.: Statistical Theory of Communication, John Wiley \& Sons Inc., New York, 1960. 